\title{
Lyotropic Ferronematic Liquid Crystals Based on New Ni, Cu and Zn Ionic Magnetic Fluids
}

\author{
C. Y. Matuo ${ }^{1}$, F. A. Tourinho ${ }^{2}$, M. H. Souza ${ }^{2}$, \\ J. Depeyrot ${ }^{3}$, and A. M. Figueiredo Neto ${ }^{4}$ \\ ${ }^{1}$ Departamento de Física, Universidade Federal de Santa Catarina, \\ Caixa Postal 476, 88040-900, SC, Brazil. \\ ${ }^{2}$ Instituto de Química, Universidade de Brasília, \\ Caixa Postal 04478, 70919-970, Brasília, DF, Brazil. \\ ${ }^{3}$ Instituto de Física, Universidade de Brasília, \\ Caixa Postal 04455, 70919-970, Brasília, DF, Brazil. \\ ${ }^{4}$ Instituto de Física, Universidade de São Paulo, \\ Caixa Postal 66318, 05315-970, São Paulo, SP, Brazil
}

Received on 26 November, 2001

\begin{abstract}
The properties of lyotropic ferronematic liquid crystals based on new $\mathrm{Ni}, \mathrm{Cu}$ and $\mathrm{Zn}$ ionic magnetic fluids are discussed. The efficiency of these new ferrofluids in the lyotropic nematic liquid crystal doping is verified and compared with the conventional surfacted ferrofluid lyotropic doping. It was observed that the structural characteristics of the lyotropics and ferrofluids determine the good formation of the ferronematics.
\end{abstract}

\section{Introduction}

Liquid crystals can be oriented [1] by electric and magnetic fields or by shear stress. Since the anisotropic part of the diamagnetic susceptibility of these materials is small, high magnetic fields, of about $10 \mathrm{kG}$, are necessary to orient the liquid crystals. In 1970, Brochard and de Gennes [2] proposed a theory of magnetic suspensions in which liquid crystals could be doped with small magnetic grains. The coupling between the magnetic grains and the liquid crystalline matrix is mainly from mechanical origin and this coupling is responsible for the orientation process [2]. The doping of liquid crystals with ferrofluids [3, 4] (above a critical concentration) reduces the magnetic field required to orient the liquid crystals by a factor $10^{3}$.

Ferrofluids or magnetic fluids are colloidal suspensions of small magnetic grains (typical dimension 10 $\mathrm{nm}$ ) dispersed in a liquid carrier. Two different types of ferrofluids are available: surfacted and ionic ferrofluid. The last one is also named electric double layered magnetic fluids (EDL-MF) [5]. In the surfacted ferrofluids, the grains (usually $\mathrm{Fe}_{3} \mathrm{O}_{4}$ ) are coated with surfactant agents (amphiphilic molecules) to prevent their flocculation (steric repulsion: physical barrier) [4]. In polar medium (water), two layers of surfactant are needed to form an external hidrophilic layer. In ionic ferrofluids, the magnetic grains (usually $\gamma-\mathrm{Fe}_{2} \mathrm{O}_{3} ; \mathrm{MnFe}_{2} \mathrm{O}_{4}$; $\mathrm{CoFe}_{2} \mathrm{O}_{4}$ ) are electrically charged to keep the colloid stable (electric repulsion) [6, 7]. The ionic citrated maghemite $\left(\gamma-\mathrm{Fe}_{2} \mathrm{O}_{3}\right)$ has both characteristics (steric and electrostatic repulsion) to prevent the aggregation of the magnetic grains. When a ferrofluid is highly diluted in a solvent, it became unstable and the grains flocculate.

Nematic liquid crystals doped with ferrofluids have been called [2] ferronematic liquid crystals. Following de Gennes' predictions, many experiments were performed with thermotropic and lyotropic ferronematic and ferrocholesteric (cholesteric liquid crystal doped with ferrofluid) liquid crystals. The attainment of a thermotropic ferronematic is a very delicate task, due to the low solubility of the magnetic fluids in the liquid crystalline matrix. Usually, the liquid crystal and the magnetic fluid separate and a homogeneous solution (as a function of time) is not achieved. The first successful observation of the macroscopic collective behavior of a magnetically doped nematic liquid crystal was obtained in 1983 by Chen and Amer [8]. In their exper- 
iment, however, the magnetic grains were much bigger than that present in actual ferrofluids: they used magnetic grains with typical dimensions of microns. On the other hand, the doping of lyotropic liquid crystals (mixture of amphiphilic molecules and a solvent, usually water [1]) with water base surfacted ferrofluids was done for the first time in 1979 by Liébert and Martinet [9], and since then this method has been used [10-14] to investigate the physical-chemical properties of lyotropics. In the most of these experiments the lyotropic liquid crystals were doped with a water base surfacted ferrofluid from Ferrofluidics Corp..

Collective behavior of the liquid crystalline matrix was observed with magnetic fields of about $5 \mathrm{G}$. The aggregation of the grains, in the presence of magnetic fields, forming needles of about $10 \mu \mathrm{m}$ long was also observed [10]. Depletion layers, i.e., regions where the magnetic grains are segregated due to topological configurations of the director, [2] were observed [10]. Following de Gennes' prediction, the minimum concentrations $\left(C_{m}\right)$ of ferrofluid magnetic grains required to orient nematic liquid crystals were determined in samples doped with surfacted ferrofluid [11] and ionic ferrofluid of $\mathrm{CoFe}_{2} \mathrm{O}_{4}$ [15]. The knowledge of $C_{m}$ is important in practical experiments in order to preserve most of the physical properties of the liquid crystalline systems.

Dynamic processes in lyotropic ferronematics were also studied [16, 17] using pulsed magnetic fields. In these studies, it was verified that there is a different dynamic behavior between ferronematics and usual nematics when samples are subjected to magnetic fields. In usual nematics the relaxation time is proportional to $H^{-2}$ [18] and in ferronematics the relaxation time is proportional to $H^{-1}$.

Due to the new features of ferronematics, mainly their remarkable response to low magnetic fields, after Brochard and de Gennes' paper [2], other theoretical approaches to the field-director coupling problem were done [19, 20, 21].

Since ferronematics (and ferrocholesterics) constitute a new class of complex magnetic fluids with academic and technological interests, it is important to know what type of ferrofluid is more efficient to produce ferronematics. The type of ferrofluid used in the doping process depends on the physics we intend to study: low or high magnetic field-director coupling, slow or fast response to external agents, and so on. Recently new ionic ferrofluids (EDL-MF) based on $\mathrm{Ni}, \mathrm{Cu}$ and $\mathrm{Zn}$ ferrites were synthesized [5, 22].

In this paper we discuss the doping of a lyotropic nematic liquid crystal with these new ferrofluids. The properties of these new ferronematics are compared with the one of usual lyotropic ferronematics obtained using different types of ferrofluids. The efficiency of each of these new ferrofluids in constituting a ferronematic is discussed.

\section{Experimental section}

\section{II.1 Materials}

The lyotropic nematic liquid crystal used is a mixture of potassium laurate (28.74 weight\%), decanol (6.64 wt\%) and water (64.62 wt\%), with an uniaxial calamitic nematic phase $N_{C}$ [23] between 12 and $35^{\circ} \mathrm{C}$. The experiments are performed at $22^{\circ} \mathrm{C}$. The nematic phase is identified by means of optical microscopy, conoscopy and X-ray diffraction.

The ionic water based ferrofluids used for the doping have magnetic grains with a mean diameter of $8.4 \mathrm{~nm}$ $\left(\mathrm{CuFe}_{2} \mathrm{O}_{4}\right), 4.4 \mathrm{~nm}\left(\mathrm{NiFe}_{2} \mathrm{O}_{4}\right)$ and $6.3 \mathrm{~nm}\left(\mathrm{ZnFe}_{2} \mathrm{O}_{4}\right)$, and magnetization at saturation of $135 \mathrm{G}, 279 \mathrm{G}$ and $0 G$, respectively. The ferrofluids used were prepared by chemical synthesis process. Copper, Nickel and Zinc $[5,22]$ spinel oxide grains are prepared by condensation method from chemical reaction among aqueous solutions of metal mixtures in alkaline medium. Grain nature and size are fixed by coprecipitation step. As far as the molar ratio is concerned, the best initial condition for all sample prepared corresponds just to the ferrite stoichiometry, i.e., 0.33. In addition, the particular base which is used, the $\mathrm{pH}$ and the temperature are extremely important in the synthesis. The best temperature range for the synthesis is around $100^{\circ} \mathrm{C}$. Usually a base excess is needed due to the acidity of the initial mixture. The reagent addition procedure, including the way and speed of mixing, acts directly to determine the average value of the grain size. The fine grains are obtained by poring the mixture as quickly as possible into the base medium under vigorous stirring. Very high ionic strength, as well as base excess, are very important in the synthesis of nickel ferrite grains. Finally, after the magnetic grains being synthesized they were washed in acid medium using a ferric nitrate solution at boiling temperature in order to promote the chemical surface stabilization. Very stable and concentrated magnetic fluids are obtained, after the chemical surface stabilization step, by dispersion in acid (alkaline) medium with suitable counterions, like nitrate or perchlorate (tetramethylammonium cation). Ferrofluids used in this study are acid ferrofluids, i. e., the grains are positively charged. The characterization of the ferrofluids were done $[5,22]$ by electronic microscopy, X-ray diffraction, magnetization measurements and birefringence measurements. 
We used concentrations of magnetic grains above the minimum concentration required to orient the nematic liquid crystals $[11,15]$ in the preparation of the ferronematics. The doping was done by carefully diluting a concentrated solution of ferrofluid, which was then introduced into a given volume of nematic with a microsyringe. The fluids were mixed by smooth stirring. Following this process, without any precipitation of the magnetic grains, the ferronematic samples present $(7 \pm 1) \times 10^{13}$ grains $/ \mathrm{cm}^{3}$.

\section{II.2 Methods and techniques}

One of the techniques used is the crossed polarizers optical microscopy. The experiment consists in to observe the ferronematic samples in a polarizing light microscope. The ferronematic sample is encapsulated inside rectangular glass microslides (from Vitro Dynamics) with the following dimensions: $25 \mathrm{~mm}$ (length), $4 \mathrm{~mm}$ (width) and $400 \mu \mathrm{m}$ (thickness). The samples are observed before and after the application of a magnetic field, and the textures are recorded with a CCD camera coupled in the microscope. A magnetic field of about $200 G$ can be applied to the sample on the microscope stage. In the case of $N_{C}$ samples, the director (optical axis) orients parallel to the applied magnetic field [12]. The microscope stage allows the observation of the ferronematic texture with different relative orientations of the director with respect to the light polarizing direction. This technique allows to check the efficiency of the ferrofluid doping when the sample is subjected to a magnetic field and also the eventual presence of clusters of magnetic grains, indicating a spurious agglomeration process.

Another optical technique used is the measurement of the transmittance as a function of the time with samples subjected to magnetic fields. The setup consists of a polarized HeNe cw laser $(10 \mathrm{~mW})$, two sources of magnetic fields, an analyzer and a photodetector connected to a computer. Figure 1 shows a sketch of the setup. The sample holders used are microslides, with the same features presented above. The laser beam direction is parallel to the $z$ axis (normal to the biggest flat surface of the microslide) and its waist at the sample's position in about $1 \mathrm{~mm}$. The magnetic field $\mathbf{H}$ is a superposition of two independent fields: a static and homogeneous field $\left(\mathbf{H}_{1}\right.$ oriented along the $y$ axis) generated by an electromagnet $\left(10 \leq H_{1} \leq 3000 \mathrm{G}\right)$, and another magnetic field $\mathbf{H}_{2}$ (oriented along the $x$ axis) is a pulsed field (strength is between 0 and $550 \mathrm{G}$ ) generated by two Helmholtz coils. $\mathbf{H}_{1}$ is oriented parallel to the longest axis of the sample holder and the direction of the analyzer. A pulse generator (square wave) controls the strength and the time interval of the pulse.

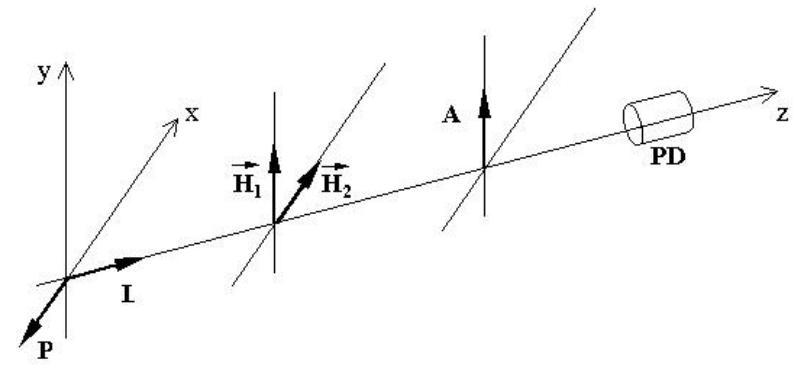

Figure 1. Sketch of the setup used to measure the optical transmittance of the ferronematic samples subjected to time dependent magnetic fields. $P, A, L$, and $P D$ are the polarizer, analyzer, laser beam directions, and photodetector, respectively. $x, y$, and $z$ are the laboratory frame axes.

\section{Results}

The lyotropic mixture doped with the water based $\mathrm{CuFe}_{2} \mathrm{O}_{4}$ ionic ferrofluid did not accept well the doping. At a concentration of $C_{o}=(7 \pm 1) \times 10^{13}$ grains $/ \mathrm{cm}^{3}$ $\left(C_{o} \gg C_{m} \sim 10^{8}\right.$ grains $\left./ \mathrm{cm}^{3}\right)$ it was observed a precipitation of the grains. Due to an agglomeration process which takes place, the actual concentration of grains in the solution is different from the initial one. The final solution presented a homogeneous brown color. With $C \sim C_{m}$ the sample is transparent and no large (micron size) clusters are observed. This fact indicates that at the actual sample $C_{m}<C<C_{o}$. The value of $C$ can be estimated assuming that, in the worst situation, $50 \%$ of the grains aggregate and, in this situation, $C \sim 3 \times 10^{13}$ grains $/ \mathrm{cm}^{3}$. At the presence of a magnetic field of $200 \mathrm{G}$ it was verified the formation of great magnetic grain clusters with about $10^{9}$ grains each (Fig. 2) . Comparing these results with those of the same nematic sample doped with a water based surfacted ferrofluid $\left(\mathrm{Fe}_{3} \mathrm{O}_{4}\right.$ magnetic grains of $10 \mathrm{~nm}$ of mean diameter and magnetization at saturation of $100 \mathrm{G}$ ), a big difference exists. It was verified that the surfacted ferrofluid diluted easily in the lyotropic liquid crystal. Contrarily to the case of the $\mathrm{CuFe}_{2} \mathrm{O}_{4}$ ionic ferrofluid, grain clusters were not observed. The response to magnetic field is fast and efficient. This response can be evaluated in Figs. 3 (surfacted ferronematic without the magnetic field) and 4 (same sample 5 seconds after the application of a $200 \mathrm{G}$ magnetic field). In the presence of the magnetic field, sample tends to orient in a planar geometry, with the director parallel to the field. The parallel lines observed in Fig. 4 are walls parallel to the applied magnetic field. The same type of dynamic observation done with $\mathrm{CuFe}_{2} \mathrm{O}_{4}$ ionic ferronematic sample indicated that the orientation of this ferronematic (in the presence of the magnetic field) takes much more time (about some hours) when compared to the surfacted ferronematic sample to achieve 
the same degree of planar orientation. Figures 5 and 6 show the $\mathrm{CuFe}_{2} \mathrm{O}_{4}$ ionic ferronematic sample without the field and $10 \mathrm{~min}$ after the application of the magnetic field. Observing these figures, it is possible to verify that there are not significant modifications in the ferronematic texture. Nevertheless, it was verified a small change in the ferronematic texture in the vicinity of the sample holder borders. This indicates that although the efficiency of $\mathrm{CuFe}_{2} \mathrm{O}_{4}$ ferrofluid is not very good to orient lyotropic liquid crystals, it is possible to orient them, but a long time is required if compared to the surfacted ferronematic results.

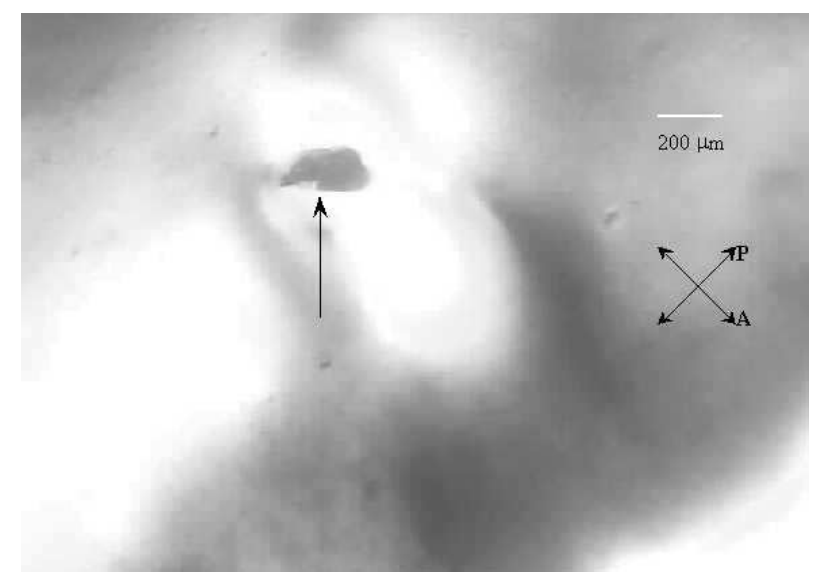

Figure 2. Ferronematic sample of $\mathrm{CuFe}_{2} \mathrm{O}_{4}$ placed in a microslide $400 \mu \mathrm{m}$ thick, subjected to a magnetic field of $200 \mathrm{G}$, observed in a polarized light microscope. The magnetic grain cluster is indicated by the arrow.

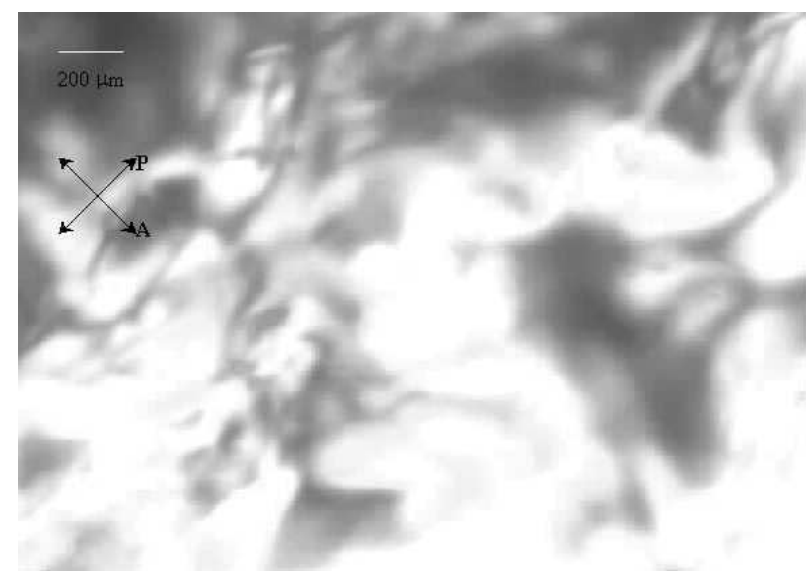

Figure 3. Sample of surfacted ferronematic in microslide $400 \mu \mathrm{m}$ thick, observed in a polarized light microscope, without the magnetic field.

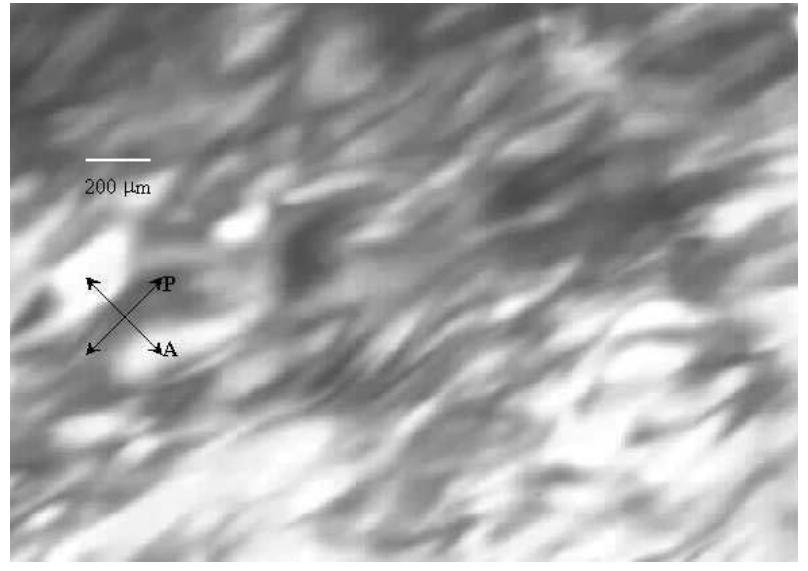

Figure 4. Sample of surfacted ferronematic in microslide $400 \mu \mathrm{m}$ thick, observed in a polarized light microscope, in the presence of a magnetic field of $200 \mathrm{G}$. The texture was obtained $5 \mathrm{~s}$ after the application of the magnetic field.

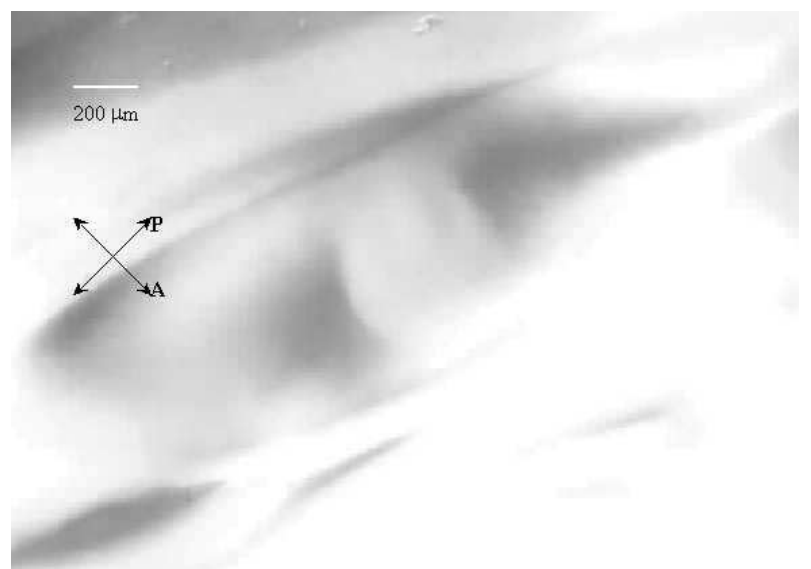

Figure 5. Sample of $\mathrm{CuFe}_{2} \mathrm{O}_{4}$ ionic ferronematic in microslide $400 \mu \mathrm{m}$ thick, observed in a polarized light microscope, without the application of the magnetic field.

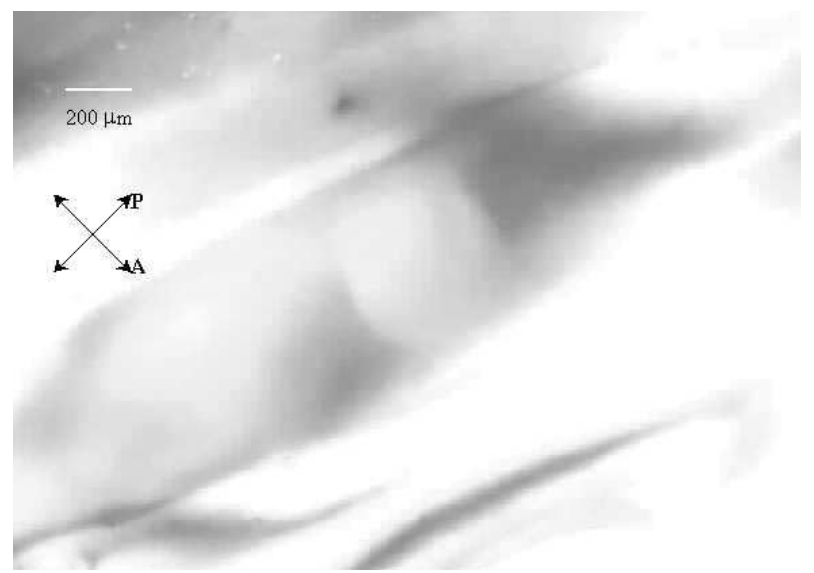

Figure 6. Sample of $\mathrm{CuFe}_{2} \mathrm{O}_{4}$ ionic ferronematic in microslide $400 \mu \mathrm{m}$ thick, observed in a polarized light microscope, in the presence of a magnetic field of $200 \mathrm{G}$ (along the horizontal direction, in the plane of the figure). The texture was obtained $10 \mathrm{~min}$ after the application of the magnetic field. 
We also studied the formation of $\mathrm{NiFe}_{2} \mathrm{O}_{4}$ and $\mathrm{ZnFe}_{2} \mathrm{O}_{4}$ based ferronematics and the same features observed in the $\mathrm{CuFe}_{2} \mathrm{O}_{4}$ ferronematic were obtained. It was verified that these ferrofluids do not dilute very well in the lyotropic liquid crystal. Some grains' aggregates with typical dimensions of microns can be observed in the texture under polarizing microscope. The actual concentration of grains can also be evaluated as done before and one has $C \sim 3 \times 10^{13}$ grains $/ \mathrm{cm}^{3}$. The overall ferronematics present a brown homogeneous color, indicating that there are some individual grains in the liquid crystal matrix. Analyzing the behavior of these ferronematics subjected to low (of the order of $200 \mathrm{G}$ ) magnetic fields, we verify that significant changes do not appear in the ferronematics texture in times of the order of a few minutes. Also in this case the walls were formed in the texture when the sample is subjected to the magnetic field.

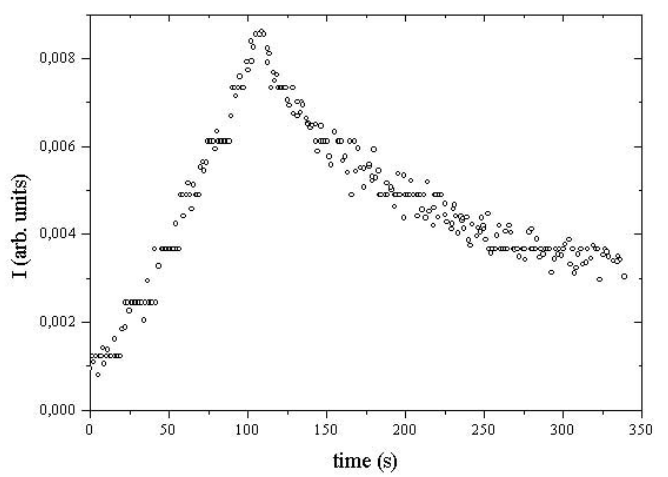

Figure 7. Optical transmittance as a function of the time. Sample of ferronematic of $\mathrm{ZnFe}_{2} \mathrm{O}_{4}$ placed in a microslide $400 \mu \mathrm{m}$ thick. Time interval of the pulse $\Delta t=100 \mathrm{~s}$.

The response time of the $\mathrm{ZnFe}_{2} \mathrm{O}_{4}$ ferronematic sample to large magnetic fields was quantitatively investigated. Due to the similarities between the $\mathrm{Zn}$ and $\mathrm{Ni}$ based ferronematics behaviors in the presence of small magnetic fields it is expected that the results obtained for the $\mathrm{Zn}$ based one can be extended to the $\mathrm{Ni}$ based ferronematic. The experimental setup used [17] is sketched in the Fig. 1. A constant magnetic field $H_{1}=2750 \mathrm{G}$ and a pulsed one (during an interval $(\Delta t)$ of about $100 \mathrm{~s}) H_{2}=550 \mathrm{G}$ are applied to the sample. Figure 7 shows the optical transmittance as a function of the time. It is possible to see that even after $100 \mathrm{~s}$ the texture did not achieve a stationary configuration. The transmittance presents an almost linear increase as a function of the time, indicating a weak mechanical coupling of the director to the magnetic grains. As the mechanical coupling strongly depends on the shape anisotropy of the grains (or even small clusters of grains), it is possible that this weak response of the nematic matrix to the magnetic field (which controls the orientation of the grains magnetic moment and, conse- quently, the grains orientation in the nematic matrix) is due to the presence of almost spherical grains (or grains clusters). After $250 \mathrm{~s}$ the switching off of the pulsed magnetic field, the optical transmittance did not return to its initial value. In the case of surfacted ferronematics, in the same conditions, the relaxation time is about $90 \mathrm{~s}$ [17]. In the ionic ferronematic sample studied here, the relaxation time calculated is $(191 \pm 3) \mathrm{s}$. For time intervals $\Delta t<90 \mathrm{~s}$, during which $\mathrm{H}_{2}$ is present, the transmittance does significantly change, within our experimental accuracy.

As a conclusion, due to this weak mechanical coupling between the nematic director and the grains, the $\mathrm{MFe}_{2} \mathrm{O}_{4}\left(\mathrm{M}^{2+}=\mathrm{Cu}, \mathrm{Ni}\right.$ and $\left.\mathrm{Zn}\right)$ based ferronematics respond very badly to small magnetic fields. The mechanical coupling does not depend on the size of the magnetic grains but depends on the grain's shape anisotropy [2]. In the electron microscopy characterization of the grains [5], they appear roughly spherical. A small aggregation (dimers or trimers) could favour the mechanical coupling of the grains with the nematic liquid crystal, but this seems to be not the case with the ionic ferrofluids studied here. This results are significantly different from the one obtained with surfacted and citrated maghemite $\left(\gamma-\mathrm{Fe}_{2} \mathrm{O}_{3}\right)$ ionic ferrofluids [17]. In that cases, it was verified that the two types of ferrofluid are efficient to orient the nematic liquid crystals. It was also verified that the surfacted ferronematic responds to the magnetic field faster than the citrated ferronematic. The differences observed in the surfacted and ionic ferronematics could indicate that the amphiphilic molecules of the surfacted ferrofluid are important in the stability of the lyotropic ferronematics. The basic units of the lyotropic liquid crystals are micelles (aggregates of amphiphilic molecules). Besides the micelles, isolated amphiphilic molecules exist in the bulk of the nematic phase. As the grains in surfacted ferrofluids are already coated with molecules having the same characteristics of the lyotropic amphiphilic molecules, the magnetic grains could be covered by them, increasing the stability of the ferronematic system. With the ionic magnetic grains this situation does not happen and could explain why both systems behaves differently in the nematic matrix. Our experience with ferronematics (ionic and surfacted) is that they remain stable for several years (at least three years old samples presents the same physical-chemical properties than fresh ones. Based on this fact we believe that there are not chemical reactions between grains and micelles taking place in ferronematics.

\section{Acknowledgments}

Fundação de Amparo à Pesquisa do Estado de São Paulo (Brazil), Conselho Nacional de Desenvolvimento Científico e Tecnológico (Brazil) and PRONEX (Brazil) are acknowledged for financial support. 


\section{References}

[1] P. G. de Gennes and J. Prost, The Physics of Liquid Crystals, $2^{\text {nd }}$ ed. (Clarendon Press, Oxford, 1993)

[2] F. Brochard and P. G. de Gennes, J. Physique 31, 691 (1970).

[3] R. E. Rosensweig, Ferrohydrodynamics (Cambridge University Press, Cambridge, 1985).

[4] S. W. Charles and J. Popplewell, in Ferromagnetic Material, edited by E. P. Wohfarth (North-Holland Publishing Company, Amsterdam, 1980), Vol. 2.

[5] F. A. Tourinho, J. Depeyrot, G. J. da Silva, and M. C. L. Lara, Braz. J. Phys. 28 (4), 413 (1998).

[6] R. Massart, US Patent No. 4329241 (May 1982).

[7] R. Massart, IEEE Trans. Mag. MAG-17, 1247 (1981).

[8] S. H. Chen and N. M. Amer, Phys. Rev. Lett. 51, 2298 (1983).

[9] L. Liébert and A. Martinet, J. Physique Lett. 40, L-363 (1979).

[10] L. Liébert and A. M. Figueiredo Neto, J. Physique Lett. 45, L-173 (1884).

[11] A. M. Figueiredo Neto and M. M. F. Saba, Phys. Rev. A 34, 3483 (1986).

[12] A. M. Figueiredo Neto, Y. Galerne, A. M. Levelut, and L. Liébert, in Physics of Complex and Supermolecular Fluids, edited by S. Safran and N. A. Clark, EXXON Monograph Series (Wiley, New York, 1987), p.347.
[13] A. M. Figueiredo Neto, Y. Galerne, A. M Levelut, and L. Liébert, J. Physique Lett. 46, L-499 (1985).

[14] A. M. Figueiredo Neto, in Phase Transitions in Complex Fluids, edited by P. Tolédano and A. M. Figueiredo Neto (World Scientific, Singapore, 1998), p.175.

[15] C. Y. Matuo, F. A. Tourinho, and A. M. Figueiredo Neto, J. Magn. Magn. Mater. 122, 53 (1993).

[16] J. C. Bacri and A. M. Figueiredo Neto, Phys. Rev. E 50, 3860 (1994).

[17] C. Y. Matuo and A. M. Figueiredo Neto, Phys. Rev. E 60, number 2, 1815 (1999).

[18] E. A. Oliveira, A. M. Figueiredo Neto, and G. Durand, Phys. Rev. A 39, R825 (1991).

[19] S. V. Burylov and Y. L. Raikher, Mol. Cryst. Liq. Cryst. 258, 107 (1995).

[20] Y. L. Raikher and V. I. Stepanov, J. Intel. Mat. Syst. Str. 7, 550 (1996).

[21] A. Yu. Zubarev and L. Yu. Iskakova, Physica A 229, 203 (1996).

[22] F. A. Tourinho, P. C. Morais, M. H. Sousa, and L. G. Macedo, Proc. 3rd Internat. Conf. on Intelligent $M a-$ terials, edited by P. F. Gobin and J. Tatibouet (The International Society for Optical Engineering, Bellingham, Washington-USA), 317 (1996).

[23] A. M. Figueiredo Neto, L. Liébert, and Y. Galerne, J. Phys. Chem. 89, 3737 (1985). 